\title{
Investigation of Bi Segregation of Cu Bicrystal Boundaries Using Aberration-Corrected STEM Depth Sectioning
}

\author{
C. A. Wade, M. Watanabe \\ Materials Science and Engineering Department Lehigh University, Bethlehem, PA
}

The $\mathrm{Cu}-\mathrm{Bi}$ system exhibits nearly no solubility of $\mathrm{Bi}$ in $\mathrm{Cu}$ along with the highest solute boundary enrichment factor among metal systems making the $\mathrm{Cu}$-Bi system ideal for segregation studies [1]. $\mathrm{Cu}-\mathrm{Bi}$ also shows a high degree of segregation induced grain boundary embrittlement. The precise cause of this embrittlement has been attributed to $\mathrm{Bi}$ size effects along the $\mathrm{Cu}$ boundary or modification of the electronic states at the boundary resulting in differences in $\mathrm{Cu}-\mathrm{Cu}$ bonds, though the exact cause is not agreed upon [2,3]. High-angle annular dark-field Scanning Transmission Electron Microscopy (HAADF-STEM) imaging has reviled the presence of possible Bi bilayer formation along $\mathrm{Cu}$ grain boundaries. Segregation behavior similar to that present in the $\mathrm{Cu}-\mathrm{Bi}$ system has been observed in the $\mathrm{Ni}-\mathrm{Bi}$ system and HAADF-STEM imaging of these $\mathrm{Ni}$ boundaries was interpreted as Bi bilayer formation along the grain interfaces [4]. To further investigate the cause of this embrittlement and to verify $\mathrm{Bi}$ bilayer formation on $\mathrm{Cu}$ grain boundaries, an accurate 3-dimensional representation of the boundary and its atomic structure must be characterized. In this study the atomic structure of the boundary has been investigated using a JEOL JEM-ARM200CF aberration-corrected STEM. This instrument was used to perform atomic resolution depth sectioning of a Bi-doped Cu bicrystal boundary to reveal the position of $\mathrm{Bi}$ atoms through the thickness of the specimen.

The advent of aberration-corrected STEM has greatly improved spatial resolution in transmission electron microscopes allowing imaging, in some cases, of atomic columns separated by as few as $47 \mathrm{pm}$ [5]. As the correctable probe-forming angle of electron probes has increased the use of larger condenser apertures has become possible. Larger condenser apertures increase the probe-forming angle causing the depth of focus to be greatly reduced [6]. By systematically varying the strength of the objective lens (focus) it is possible to section through the thickness of a specimen obtaining an image at each focal step. This collection of images can be carried out by in-house scripts in the Gatan DigitalMicrogrph platform to create a 3-dimensional image stack with the depth resolution approaching 8-7 nm for point like objects. Previous work using STEM depth sectioning has focused on individual Bi atoms in bulk Si and segregation along ceramic interfaces [6]. In this study the STEM depth sectioning technique has been applied to a metallic system to help interpret the distribution of the segregant elements along and through the grain boundary.

For these depth sectioning experiments $\mathrm{Cu}$ twist-bicrystals with a nominal misorientation of approximately $33^{\circ}$ about the [001] axis were used. The bicrystals were doped with Bi by being held in contact with $\mathrm{Bi}$ shot in an inert atmosphere tube furnace at $600{ }^{\circ} \mathrm{C}$ for 72 hours. The doped samples were metallographically prepared and coated with a $5 \mathrm{~nm}$ Ir film prior to Focused Ion Beam (FIB) milling. Thin-foil specimen preparation was performed on a FEI DB-235 FIB instrument where the specimens were mounted on Mo grids and thinned to electron transparency. To reduce the effects of ion beam damage and surface redeposition through the FIB process a Fischione 1040 Nanomill was used as the final specimen preparation step. Prior to depth sectioning the quantitative X-ray energy dispersive spectrometry (XEDS) was performed to confirm both the presence and location of Bi along the bicrystal boundaries using the JEOL JEM-200CF at 80 and $200 \mathrm{kV}$. For this quantification $\zeta$-factor analysis was 
employed to determine both boundary coverage $\left(\Gamma^{\mathrm{ex}}\right)$ and thickness profiles across the Bi rich boundary facets of the specimens [7]. Depth sectioning was performed over the boundary regions at $200 \mathrm{kV}$ with a probe-forming angle of approximately $37 \mathrm{mrad}$. Depth sectioning of the boundary revealed segregation away from the interior of the specimen as well as localization of segregants to dislocations when present along boundary. The DF-STEM images in Figure 1 show segregation of $\mathrm{Bi}$ to the top and bottom surfaces a specimen thats bicrystal boundary has been slightly inclined with respect to the optic axis allowing for a larger boundary projection width. Atomic columns of $\mathrm{Bi}$ are visible in Figure 1a showing the presence of $\mathrm{Bi}$ on the top surface of the specimen. The presence of $\mathrm{Bi}$ on the lower surface of the specimen is shown in Figure $1 \mathrm{~b}$ where the bottom surface of the specimen is nearly infocus allowing the $\mathrm{Bi}$ towards this surface to become more sharply defined and have greater contrast. The lack of contrast along the boundary between the two surfaces as the focal position of the probe is moved though the specimen indicates non-homogeneity in the distribution of Bi through the thickness of the specimen.

[1] M.P. Seah and E.D. Hondros, Proc. Roy. Soc. London A, Math. Phys. Sci. 335 (1973), 191.

[2] R. Schweinfest, et al., Nature 432 (2004), 1008.

[3] G. Duscher et al, Nature Materials 3 (2004), 621.

[4] J. Lou et al., Science 333 (2011), 1730.

[5] H. Sawada et al., J. Electron Microsc. 58 (2009), 357.

[6] A. Borisevich, et al., Proc. National Academy Sci. 103 (2006), 3044.

[7] M. Watanabe and D.B. Williams, J. Microsc. 221 (2006), 89.

[8] The authors wish to acknowledge financial support from the NSF through grants DMR-0804528 and DMR-1040229.

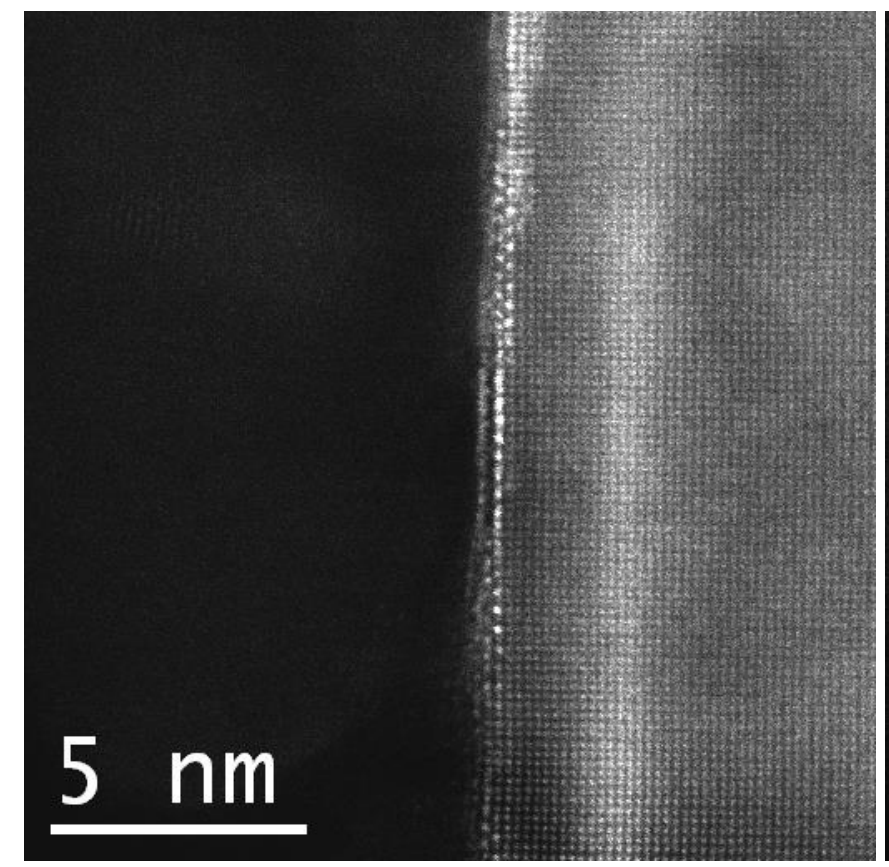

(a)

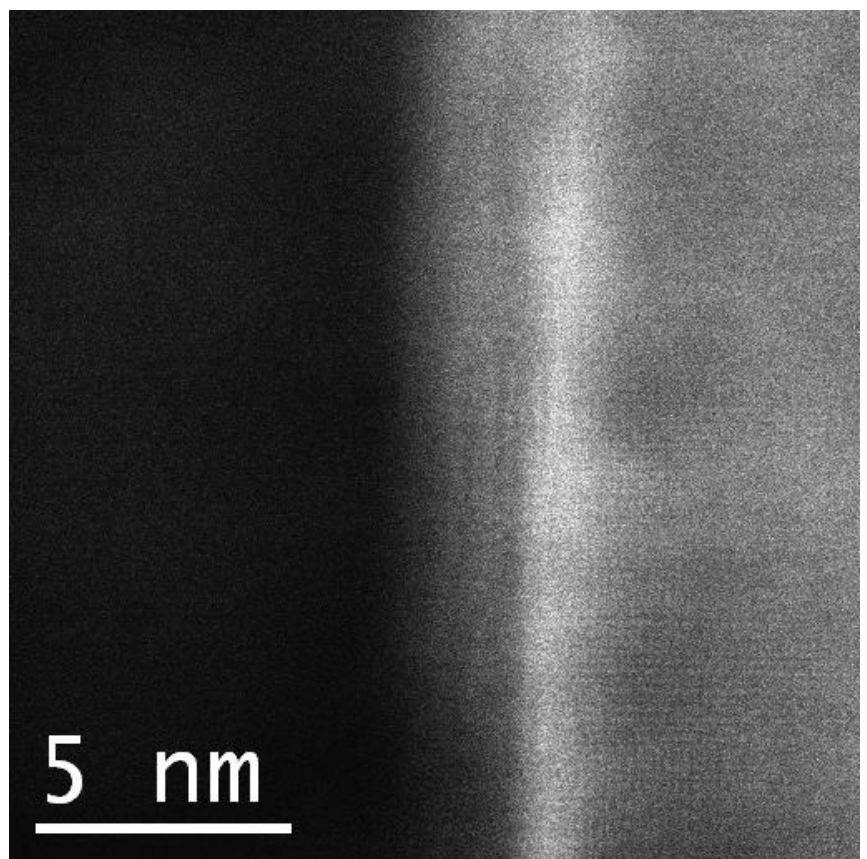

(b)

Figure 1 - An inclined Bi-doped $\mathrm{Cu} 33^{\circ}$ twist-bicrystal boundary viewed by HAADF-STEM imaging with an electron probe focused on (a) the top surface and (b) the bottom surface of the specimen 